\title{
The importance of sustained attention in early Alzheimer's disease
}

\author{
Jonathan D Huntley PhD. ${ }^{1}$, Adam Hampshire PhD², Daniel Bor PhD², \\ Adrian M Owen $\mathrm{PhD}^{4}$, Robert J Howard MD. ${ }^{1}$
}

${ }^{1}$ Institute of Psychiatry, Psychology and Neuroscience, King's College London, UK. Tel: 44 2078480508. Email: jonathan.huntley@kcl.ac.uk (corresponding author). 2Division of Brain Sciences, Imperial College London, UK; ${ }^{3}$ Sackler Centre for Consciousness Science, University of Sussex, UK, ${ }^{4}$ Brain and Mind Institute, University of Western Ontario, Canada

KEYWORDS: Alzheimer's disease, cognition, attention, neuropsychology KEY POINTS:

1) Patients with early Alzheimer's disease have significant deficits in sustained Attention, as measured using the sustained attention to response task (SART). 2) Sustained attention impairment significantly predicts performance on general cognitive testing.

3) Deficits in sustained attention should therefore be taken into account during clinical assessment and everyday management of individuals with early AD.

This work was supported by the Medical research Council (J.D.H grant number G0901982).

Word count: 3553 


\section{ABSTRACT}

Introduction: There is conflicting evidence regarding impairment of sustained attention in early Alzheimer's disease (AD). We examine whether sustained attention is impaired and predicts deficits in other cognitive domains in early AD.

Methods: 51 patients with early AD (MMSE > 18) and 15 healthy elderly controls were recruited. The sustained attention to response task (SART) was used to assess sustained attention. A subset of 25 patients also performed tasks assessing general cognitive function (ADAS-Cog), episodic memory (Logical memory scale, Paired Associates Learning), executive function (verbal fluency, grammatical reasoning) and working memory (digit and spatial span).

Results: AD patients were significantly impaired on the SART compared to healthy controls (total error $\beta=19.75, p=0.027$ ). SART errors significantly correlated with MMSE score (Spearman's rho $=-0.338, p=0.015)$, and significantly predicted deficits in ADAS-Cog $(\beta=0.14, p=0.004)$.

Discussions: Patients with early AD have significant deficits in sustained attention, as measured using the SART. This may impair performance on general cognitive testing, and therefore should be taken into account during clinical assessment, and everyday management of individuals with early $A D$. 


\section{INTRODUCTION}

Alzheimer's disease (AD) is the commonest form of dementia, affecting approximately 44.4 million people worldwide (Prince, et al. 2013). The early stages of $A D$ are associated primarily with episodic memory impairment (Dubois, et al. 2007). However, deficits in other cognitive domains, including attention, working memory and executive function may also be present at the earliest clinical stages of the disease (Huntley and Howard 2010; Perry and Hodges 1999). Attentional deficits may be overlooked in early $A D$, and presumed deficits in other cognitive domains may be secondary to underlying attentional deficits, including basic processes such as sustained attention (Parasuraman and Haxby 1993). Sustained attention refers to the ability to maintain or focus attention over a period of time (Lezak, et al. 2012). This capacity is differentiated from selective attention, the ability to focus on relevant stimuli while ignoring irrelevant or distracting stimuli, and divided attention, the ability to focus on 'more than one relevant stimulus or process at one time' (Lezak et al. 2012; Perry and Hodges 1999). Recent studies have provided evidence that sustained attention can be dissociated from other attentional subsystems such as attentional selectivity and capacity (McAvinue, et al. 2012).

There is considerable overlap between cognitive models of attention and those of working memory and executive function (Baddeley 1992). Whilst in early AD there is evidence of impairment in the more executively demanding processes of selective and divided attention (Huntley and Howard 2010; Perry and Hodges 1999), the evidence for impairment of sustained attention is so far inconclusive. Initial studies suggested that patients with mild dementia performed normally on tasks assessing sustained attention (Lines, et al. 1991; Perry and Hodges 1999). Subsequent investigations however, have suggested that sustained attention is impaired in mild 
AD (Rizzo, et al. 2000), but is preserved in earlier or 'minimal' AD (Baudic, et al. 2006; Perry, et al. 2000). Investigations in patients with mild cognitive impairment $(\mathrm{MCl})$ have also revealed evidence of impairment in sustained attention, with decline in sustained attention in $\mathrm{MCl}$ suggested by one study as an early indicator of transition from MCI to dementia (Saunders and Summers 2011).

One reason for this discrepancy in the literature relates to difficulties in assessing sustained attention. Sustained attention is traditionally investigated using continuous performance tests (CPT). Participants are required to sustain attention or vigilance over long sequences of non-target stimuli and respond only when infrequent targets were presented. In these tasks, sustained attention is assessed by 'overall vigilance' (overall performance on the task), and the 'vigilance decrement' which refers to either a decline in the detection of target stimuli over time, or an increase in reaction time to response over the length of the task (Sarter, et al. 2001). In order to avoid potential ceiling effects with both healthy older people and AD participants, variations of this task have used perceptual degradation of stimuli. These strategies, however, have failed to completely overcome ceiling effects seen in early AD (Berardi, et al. 2005; Parasuraman and Haxby 1993). In comparison, sustained attention can be assessed by tasks such as the sustained attention to response task (SART) (Robertson, et al. 1997). In this variation on the CPT, participants are required to respond to regular non targets, and withhold their automatic responses to infrequent targets (Robertson et al. 1997). Correct performance of the SART therefore requires participants to sustain sufficient attention so that when a target digit appears, they are able to inhibit their response and substitute it with the directly antagonistic action of withholding a response (Manly, et al. 1999). As errors on the SART task occur quickly, impairment in sustained attention is reflected in the total number of errors 
made, rather than the change in performance over time. Previous studies have demonstrated that prior to making an error there is an acceleration of reaction time to non-targets, which has been interpreted as an increasingly automatic response to non-target stimuli due to the reduction of sustained attention to task material (Manly et al. 1999; Robertson et al. 1997) (See online supplementary materials for further discussion of the theoretical basis for impairment on vigilance and SART tasks). In addition it is argued that as the SART requires the inhibiton of an automatic response on rare, unpredictable occasions, it more accurately reflects real life situations and is more sensitive to sustained attention deficits than traditional vigilance tasks (Staub, et al. 2013). Previous studies in participants with brain injuries and healthy young participants have demonstrated that the SART is sensitive to lapses in sustained attention, and that performance correlates with everyday attentional difficulties (Robertson et al. 1997).

Given the emerging evidence for deficits in sustained attention in early AD (Baudic et al. 2006), an important question is whether deficits attributed to other cognitive domains, such as episodic memory and executive function may be confounded by impaired sustained attention. Clinically, general cognitive function is often assessed using 'bedside' tests of cognitive function such as the MMSE or ACE-R (Hsieh, et al. 2013) or through more extensive research assessments such as the ADAS-Cog (Rosen, et al. 1984), all of which require that patients sustain attention while they engage with and complete the assessment. Therefore, evidence of impaired sustained attention to tasks lasting less than a few minutes may be clinically highly important as a confounder during routine clinical assessment.

In order to assess this we examined performance on the sustained attention to response task in patients with early $A D$ and healthy control participants. We 
hypothesised that patients with early AD would be impaired on performance of the SART compared with healthy elderly controls and that SART errors would correlate with severity of general cognitive impairment as measured with the MMSE. A subset of patients with mild $A D$ was also assessed using an additional series of cognitive tasks investigating general cognitive function, working memory, episodic memory and executive function. We hypothesised that sustained attention deficits, as measured using the SART, would predict deficits in other cognitive domains in early AD. 


\section{METHODS}

\section{Participants}

The current study analyses unpublished data from participants recruited for two separate studies. Data was taken from 15 healthy elderly control participants and 26 patients with mild $A D$ recruited for a study examining working memory in early $A D$ (Huntley, et al. 2011). Data was also taken from 25 patients with mild AD recruited for a cognitive training study (Huntley, et al. 2016). A total of 15 healthy elderly participants and 51 patients with early AD were therefore included in the current study. All AD patients were recruited from community memory services of the South London and Maudsley NHS Foundation Trust. All patients had a diagnosis of possible or probable AD (according to NINCDS-ADRDA criteria (McKhann, et al. 1984). Inclusion criteria were an MMSE score of $>18 / 30$ and age $>60$ years.

Exclusion citeria included co-existent neurological or psychiatric disease, substance misuse or significant auditory or visual impairment. Diagnoses were made by experienced Old Age Psychiatrists unconnected to the study in conjunction with multi-disciplinary team assessments. Healthy elderly controls were recruited from a database of healthy elderly individuals who had provided consent to be contacted regarding clinical research. All participants had capacity to provide written informed consent to participate in the study, which was approved by the relevant national research ethics committees (REC reference numbers 08/H0809/19 and 10/H0304/68). All assessments were conducted using the same materials, by the same researcher in participants' homes. Therefore although data for the current study is taken from two separate studies, there were no significant differences between the format and method of data collection between the studies. Please see online supplementary materials for further information regarding participants. 


\section{The Sustained attention to response task}

Sustained attention was assessed using the Sustained Attention to Response Task (SART) (Robertson et al. 1997). In this task, single digit numbers (0-9) were individually and randomly presented for $500 \mathrm{~ms}$ on a computer screen. The font size and boldness of the digits varied, and a small cross was presented for $1000 \mathrm{~ms}$ between digits. All stimuli were presented in black on a white background. The total time between digit onset and digit onset was $1500 \mathrm{~ms}$. During this time, the participant was asked to respond by pressing the space bar on a keyboard in response to all frequent non-target digits (i.e. all digits other than 3), and were told not to press the bar if a non-frequent target (the number 3) was presented. All participants had a demonstration of the task by the researcher and then completed fifty practice trials. Verbal feedback was provided during the practice trails to ensure participants understood and could retain the instructions and perform the task. Following the practice trials all participants completed 270 test trials, which included 30 targets (number 3 ) and 240 non-targets (all other numbers). The targets were pseudorandomly distributed to ensure they were unpredictable but occurred with a similar frequency throughout the task (approximately 7 targets for every 65 trials). The task lasted $405 \mathrm{~s}$ in total. During the test trials, no feedback was given regarding task performance. The number of commission errors (responding when the target digit ' 3 ' was presented), omission errors (not responding to all other non-target digits) and total errors were recorded for analysis. The response time between presentation of each digit and response was also recorded, and the mean response time of the 4 trials prior to all commission errors and correct responses was calculated for analysis. 


\section{Baseline assessments and cognitive measures}

All participants had data collected on age, gender and years of education. The National Adult Reading Test (NART) (Nelson 1982), was administered as a test of pre-morbid IQ. The shortened version of the Geriatric Depression Scale (GDS-15) (Sheikh and Yesavage 1986) was used to exclude significant depressive illness (signified by a score of $>6$ ). For the healthy elderly controls and 26 AD patients taken from the working memory study, data on MMSE and working memory was available. Working memory was assessed in both verbal and spatial domains using digit (Bor, et al. 2004) and spatial span tasks (Bor, et al. 2006). For the 25 AD patients taken from the cognitive training study, data from a baseline (pre cognitive training) battery of cognitive tasks assessing episodic memory, (Logical Memory II test (Wechsler 1997) and Paired Associates Learning task (PAL) (Gould, et al. 2005); executive function (verbal fluency task (Lezak et al. 2012) and grammatical reasoning task, (Owen, et al. 2010); instrumental activities of daily living (Lawton and Brody 1969); and general cognitive function (Alzheimer's disease assessment scalecognitive section (ADAS-Cog) (Rosen et al. 1984) were also available Please see supplementary materials for further details of all tasks.

\section{Statistical analysis}

For all data the assumption of normality was assessed by plotting histograms and QQ plots of the raw data and calculating values of skewedness, kurtosis and the Kolmogorov-Smirnov test using SPSS (IBM 2013). To examine group differences in demographics, screening tasks and performance on the SART, independent T tests were conducted for normally distributed data, and medians, interquartile ranges and 
independent samples Mann Whitney $\mathrm{U}$ tests were conducted for non-normally distributed data. To assess the correlation between MMSE score and performance on the SART, Spearman's rho was calculated for MMSE score and SART errors using SPSS (IBM 2013).

As SART error data was not normally distributed, quantile regression analyses were conducted using the 'qreg' command in Stata 12. In separate analyses each of the SART outcomes were included as dependent variables with age and group as independent variables, to examine group differences in performance on the SART. In the $25 \mathrm{AD}$ participants for whom additional cognitive measures were available, scores on all available cognitive and functional tasks were included as dependent variables in separate quantile regression analyses, with SART errors and age as independent variables, using the 'qreg' command in Stata 12 (StataCorp. 2011 College Station, TX). Quantile regression estimates the median of the dependent variable, conditional on the values of the independent variables. In contrast to ordinary regression, median regression finds a line of best fit through the data that minimizes the sum of the absolute residuals rather than the sum of the squares of the residuals (StataCorp 2013). These analyses allowed regression coefficients to be calculated for each SART error (commission, omission and total errors) for each dependent cognitive outcome measure. 


\section{RESULTS}

Demographic information is displayed in Table 1. The groups were well matched for years of education, premorbid IQ and GDS scores. There was an expected significant difference between the groups in MMSE score. There was also a significant difference between the groups in age, with the AD group being older than the healthy elderly controls.

\section{Sustained attention}

Results of all SART outcomes are shown in Table 2. Examining total errors (combining both commission and omission errors), demonstrated that when age was controlled for, there was a significant group difference $(\underline{\beta}=19.75, \mathrm{SE} 8.71,95 \% \mathrm{CI}$ 2.34, 37.16, $p=0.027$ ), with control subjects overall making 8 errors, whilst AD subjects made 29 errors (see Figure 1 and Table 2).

Examining each error type separately, there was a significant difference between the groups for omission errors $(\underline{\beta}=16$, SE $6.08,95 \% \mathrm{Cl} 3.84,28.16, p=0.011)$, with control participants making only 3 errors, compared to AD participants who made 19 errors (see Table 2). There was a non-significant group difference for commission errors $(p=0.510)$, with control participants making 4 errors and AD participants making 8 errors (see Table 2). To assess if more errors were made by AD patients with time on task, the median number of errors made in the first 68 trials and last 68 trials were compared. Although more omission errors were made in the first 68 trials (median $=5(1,14)$ than in the last 68 trials (median $=4(0,8)$, this was nonsignificant (Wilcoxon signed rank test, $p=0.118$ ). There was no difference in the median number of commission errors made between the first and last blocks of 68 trials (medians $=2(0,4)$ vs $2(0,4)$ 


\section{Response times}

Controlling for age, there were no significant group differences in response times. As shown in Table 2, the mean response time for the 4 trials prior to both correct responses and commission errors was faster in control subjects than AD subjects, but not significantly.

To investigate within group differences in response times leading up to correctly withheld and incorrectly responded target trials, related samples Wilcoxon signed rank tests were conducted for each group. For control subjects there was no significant difference (474ms vs. $467 \mathrm{~ms}, p=0.245)$. However within the AD patients there was a significant difference in response times leading up to correctly withheld and incorrectly responded target trials (588ms vs. 566ms respectively, $p=0.014$ ). Therefore, although all participants demonstrated faster response times leading up to commission errors, this was only significantly different in the AD group.

\section{Correlation between MMSE and total SART errors}

As shown in Figure 2, across all AD participants, there was a significant correlation between total errors and MMSE score, (Spearman's rho $=-0.338, \mathrm{n}=51, p=$ 0.015). Examining only commission errors there is a near significant correlation between errors and MMSE (Spearman's rho $=-0.26, p=0.067$ ), and a significant correlation between omission errors and MMSE (Spearman's rho $=-0.284, \mathrm{p}=$ 0.043). All correlations are negative, indicating an increase in errors with a decrease in MMSE. 


\section{Regression analyses}

As shown in Table 3, when age was controlled for in the regression model, sustained attention errors predicted a significant proportion of the variability in performance on the ADAS-Cog tasks. For the ADAS-Cog the quantile regression coefficient $\beta=0.14$ (SE 0.04 ), $95 \% \mathrm{Cl} 0.05,0.22, p=0.004)$. Therefore for every 1 unit change in total errors, the median ADAS-Cog score will increase by 0.15 . When each error type was examined separately the regression coefficients were significant for both commission ( $\beta=0.64$ (SE 0.19, 95\% Cl 0.25, 1.04, $p=0.003$ ) and omission errors ( $\beta=0.14$ (SE 0.06), 95\% Cl 0.02, 0.26, $p=0.022$ ). For the spatial span task, the quantile regression coefficient neared significance $(\beta=-0.02$ (SE 0.01$), 95 \% \mathrm{Cl}-$ $0.04,-0.001), p=0.057)$. For instrumental activities of daily living, the regression coefficient was significant for commission errors ( $\beta=-0.11$ (SE 0.05), $95 \% \mathrm{Cl}-0.22$, $0.002, p=0.045$ ), but not for omission or total errors. Sustained attention errors had no significant effects on any of the episodic memory, executive function or verbal working memory tasks. Please see supplementary table 1 for scores on individual cognitive tasks.

\section{DISCUSSION}

This study found that patients with early AD made significantly more total errors, including significantly more omission errors, on the sustained attention to response task than healthy elderly controls. There was also a reduction in reaction time preceding a commission error being made. This suggests a combination of overall reduced sustained attention to the task and the occurrence of more attentional lapses in patients with early AD compared to healthy elderly controls. 
Whilst it is possible that errors may reflect difficulties in retaining task instructions, rather than with sustained attention in AD patients, this is unlikely as participants self-corrected and continued to correctly perform the task after errors were made. There was no evidence of an increase in errors over the course of the task indicating ongoing awareness and recall of the task instructions during the task.

There was also a significant correlation between sustained attention impairment and MMSE score, indicating impairment and deterioration of sustained attention during the earliest stages of $A D$. This is in keeping with literature that has suggested that sustained attention deficits are apparent at the earliest stages of $A D$ and may be associated with progression from MCI to dementia (Saunders and Summers 2011). The clinically important finding from our data is that impairment in sustained attention is evident during performance of a task over a short period of time. The SART took 405 seconds, less than 7 minutes, and therefore this demonstrated that patients have difficulties sustaining attention over a shorter duration than is required to complete several clinically-used assessments of general cognitive function, such as the ACE-R or ADAS-Cog. It is also notable that in the regression analysis, SART errors significantly predicted ADAS-Cog score. This needs to be interpreted with caution, as the numbers in the regression analysis were small, and regression analyses do not allow the contribution of sustained attention to be clearly disentangled from other cognitive functions. Nonetheless, it may be that sustained attention deficits impair the ability of patients at the earliest stages of $A D$ to perform general cognitive function assessments, and clinicians should be aware of this when seeking to assess cognitive domains using a general tool that may take several minutes to deliver. 
Whilst it may seem unsurprising that patients with early AD have impaired sustained attention, it is notable that until recently the literature concluded that sustained attention was intact in mild AD (Perry and Hodges 1999). The SART is a sensitive tool to elicit sustained attentional deficits and performance correlates with everyday attentional function. In our regression analysis, commission errors significantly predicted variability in scores of instrumental ADLs. There were no significant effects of omission or total errors on IADL scores and numbers were small, however the finding that commission errors are associated with impairment in everyday function, is consistent with previous studies suggesting that sustained attention impairment may affect more complex activities of daily living such as driving safety in early AD (Parasuraman and Nestor 1991). The SART may therefore be a useful clinical adjunct in the assessment of cognitive impairment, as it is easy and quick to administer and sensitive to sustained attention deficits in early AD.

The observation that sustained attention is impaired at the earliest stages of AD may also provide a target for both cognitive and pharmacological intervention. There is evidence for the efficacy of cognitive rehabilitation focusing on sustained attention in patients post acquired brain injury (Park and Ingles 2001), stroke (Loetscher and Lincoln 2013) and in ADHD in adults (O'Connell, et al. 2008). There are limited trials examining cognitive interventions focusing on attention in $A D$, however there is emerging evidence that focused working memory training can be effective (Anguera, et al. 2013) and the evidence of the efficacy of attentional training in older patients post brain injury (Park and Ingles 2001) may indicate the potential for efficacy of similar cognitive interventions in early AD.

Pharmacological interventions have also been shown to improve sustained attention in several clinical groups (Coulthard, et al. 2006; Turner, et al. 2004). Donepezil has 
been demonstrated to improve sustained attention in brain injured individuals (Zhang, et al. 2004) and in patients with Parkinson's disease dementia (Wesnes, et

al. 2005). The use of cholinesterase inhibitors to improve cognitive function in AD is well established (Birks 2006), however there is also evidence that cholinesterase inhibitors improve attention in AD (Foldi, et al. 2005). Therefore, not only are deficits in sustained attention clinically important at the earliest stages of $A D$, they may be amenable to both pharmacological and non-pharmacological interventions.

Overall this study demonstrated that patients at the earliest stages of AD have significant impairment in sustained attention. These deficits in sustained attention may impair performance on clinically used general cognitive assessments. Impairments in basic sustained attention therefore need to be taken into account during the development and delivery of cognitive assessments, and during the everyday management of individuals with early stage AD.

\section{ACKNOWLEDGEMENTS}

We are grateful to all the participants and carers involved in this study.

\section{COMPETING INTERESTS}

The authors declare no competing interests.

\section{FUNDING SOURCE}

This work was supported by the Medical research Council (J.D.H grant number G0901982). Participant recruitment was supported by the Mental Health of Older Adults and Dementia Clinical Academic Group within King's Health Partners Academic Health Sciences Centre at the Institute of Psychiatry, Psychology and 
Neuroscience, King's College London and the South London and Maudsley National Health Service Foundation Trust.

\section{ETHICAL STANDARDS}

The authors assert that all procedures contributing to this work comply with the ethical standards of the relevant national and institutional committees on human experimentation and with the Helsinki Declaration of 1975, as revised in 2008.

\section{AUTHOR CONTRIBUTIONS}

All authors contributed to the concept and design of the study. JDH collected the data, conducted the analyses and wrote the paper. $\mathrm{AO}, \mathrm{AH}$ and $\mathrm{DB}$ provided materials and contributed to the analyses, interpretation of data and discussion. RJH contributed to the design, analyses and discussion. 


\section{REFERENCES}

Anguera JA, Boccanfuso J, Rintoul JL, Al-Hashimi O, Faraji F, Janowich J, Kong E, Larraburo Y, Rolle C, Johnston E, et al. 2013 Video game training enhances cognitive control in older adults. Nature 501 97-101.

Baddeley A 1992 Working memory. Science 255 556-559.

Baudic S, Barba GD, Thibaudet MC, Smagghe A, Remy P \& Traykov L 2006 Executive function deficits in early Alzheimer's disease and their relations with episodic memory. Arch Clin Neuropsychol 21 15-21.

Berardi AM, Parasuraman R \& Haxby JV 2005 Sustained attention in mild Alzheimer's disease. Dev Neuropsycho/ 28 507-537.

Birks J 2006 Cholinesterase inhibitors for Alzheimer's disease. Cochrane Database Syst Rev CD005593.

Bor D, Cumming N, Scott CE \& Owen AM 2004 Prefrontal cortical involvement in verbal encoding strategies. The European journal of neuroscience 19 3365-3370. Bor D, Duncan J, Lee AC, Parr A \& Owen AM 2006 Frontal lobe involvement in spatial span: converging studies of normal and impaired function. Neuropsychologia 44 229-237.

Coulthard E, Singh-Curry V \& Husain M 2006 Treatment of attention deficits in neurological disorders. Curr Opin Neurol 19 613-618.

Dubois B, Feldman HH, Jacova C, Dekosky ST, Barberger-Gateau P, Cummings J, Delacourte A, Galasko D, Gauthier S, Jicha G, et al. 2007 Research criteria for the diagnosis of Alzheimer's disease: revising the NINCDS-ADRDA criteria. Lancet Neurol 6 734-746. 
Foldi NS, White RE \& Schaefer LA 2005 Detecting effects of donepezil on visual selective attention using signal detection parameters in Alzheimer's disease. Int $J$ Geriatr Psychiatry 20 485-488.

Gould RL, Brown RG, Owen AM, Bullmore ET, Williams SC \& Howard RJ 2005 Functional neuroanatomy of successful paired associate learning in Alzheimer's disease. Am J Psychiatry 162 2049-2060.

Hsieh S, Schubert S, Hoon C, Mioshi E \& Hodges JR 2013 Validation of the Addenbrooke's Cognitive Examination III in frontotemporal dementia and Alzheimer's disease. Dement Geriatr Cogn Disord 36 242-250.

Huntley J, Bor D, Hampshire A, Owen A \& Howard R 2011 Working memory task performance and chunking in early Alzheimer's disease. Br J Psychiatry 198398 403.

Huntley J, Hampshire A, Bor D, Owen AM \& Howard R 2016 Adaptive working memory strategy training in early Alzheimer's disease: a randomised controlled trial. British Journal of Psychiatry in press.

Huntley JD \& Howard RJ 2010 Working memory in early Alzheimer's disease: a neuropsychological review. Int J Geriatr Psychiatry 25 121-132.

IBM 2013 Statistical Package for Social Sciences (SPSS) version 22.0. edn 22.0. Lawton MP \& Brody EM 1969 Assessment of older people: self-maintaining and instrumental activities of daily living. Gerontologist 9 179-186.

Lezak MD, Howieson DB, Bigler ED \& Tranel D 2012 Neuropsychological assessment (5th ed.). New York: Oxford University Press.

Lines CR, Dawson C, Preston GC, Reich S, Foster C \& Traub M 1991 Memory and attention in patients with senile dementia of the Alzheimer type and in normal elderly subjects. J Clin Exp Neuropsychol 13 691-702. 
Loetscher T \& Lincoln NB 2013 Cognitive rehabilitation for attention deficits following stroke. Cochrane Database Syst Rev 5 CD002842.

Manly T, Robertson IH, Galloway M \& Hawkins K 1999 The absent mind: further investigations of sustained attention to response. Neuropsychologia 37 661-670. McAvinue LP, Habekost T, Johnson KA, Kyllingsbaek S, Vangkilde S, Bundesen C \& Robertson IH 2012 Sustained attention, attentional selectivity, and attentional capacity across the lifespan. Atten Percept Psychophys 74 1570-1582.

McKhann G, Drachman D, Folstein M, Katzman R, Price D \& Stadlan EM 1984 Clinical diagnosis of Alzheimer's disease: report of the NINCDS-ADRDA Work Group under the auspices of Department of Health and Human Services Task Force on Alzheimer's Disease. Neurology 34 939-944.

Nelson HE 1982 National Adult Reading Test. Windsor, UK: NFER-Nelson. O'Connell RG, Bellgrove MA, Dockree PM, Lau A, Fitzgerald M \& Robertson IH 2008 Self-Alert Training: volitional modulation of autonomic arousal improves sustained attention. Neuropsychologia 46 1379-1390.

Owen AM, Hampshire A, Grahn JA, Stenton R, Dajani S, Burns AS, Howard RJ \& Ballard CG 2010 Putting brain training to the test. Nature 465 775-778.

Parasuraman R \& Haxby JV 1993 Attention and Brain Function in Alzheimer's Disease: A Review. Neuropsychology 7 242-247.

Parasuraman R \& Nestor PG 1991 Attention and driving skills in aging and Alzheimer's disease. Hum Factors 33 539-557.

Park NW \& Ingles JL 2001 Effectiveness of attention rehabilitation after an acquired brain injury: a meta-analysis. Neuropsychology 15 199-210.

Perry RJ \& Hodges JR 1999 Attention and executive deficits in Alzheimer's disease. A critical review. Brain 122 ( Pt 3) 383-404. 
Perry RJ, Watson P \& Hodges JR 2000 The nature and staging of attention dysfunction in early (minimal and mild) Alzheimer's disease: relationship to episodic and semantic memory impairment. Neuropsychologia 38 252-271.

Prince M, Guerchet M, Prina M \& International AsD 2013 Policy Brief for Heads of Government: The Global Impact of Dementia 2013- 2050. Ed AsDI (ADI). London. Rizzo M, Anderson SW, Dawson J, Myers R \& Ball K 2000 Visual attention impairments in Alzheimer's disease. Neurology 54 1954-1959.

Robertson IH, Manly T, Andrade J, Baddeley BT \& Yiend J 1997 'Oops!': performance correlates of everyday attentional failures in traumatic brain injured and normal subjects. Neuropsychologia 35 747-758.

Rosen WG, Mohs RC \& Davis KL 1984 A new rating scale for Alzheimer's disease. Am J Psychiatry 141 1356-1364.

Sarter M, Givens B \& Bruno JP 2001 The cognitive neuroscience of sustained attention: where top-down meets bottom-up. Brain Res Brain Res Rev 35 146-160. Saunders NL \& Summers MJ 2011 Longitudinal deficits to attention, executive, and working memory in subtypes of mild cognitive impairment. Neuropsychology 25 237248.

Sheikh JI \& Yesavage JA 1986 Geriatric Depression Scale (GDS): recent evidence and development of a shorter

version. . Clinical Gerontology. 5 165-173.

StataCorp 2013 Stata 13 Base Reference Manual. College Station, TX: Stata Press. Staub B, Doignon-Camus N, Despres O \& Bonnefond A 2013 Sustained attention in the elderly: what do we know and what does it tell us about cognitive aging? Ageing Res Rev 12 459-468. 
Turner DC, Clark L, Dowson J, Robbins TW \& Sahakian BJ 2004 Modafinil improves cognition and response inhibition in adult attention-deficit/hyperactivity disorder. Biol Psychiatry 55 1031-1040.

Wechsler D 1997 Wechsler Memory Scale- Third Edition. San Antonio, Texas: The Psychological Corporation.

Wesnes KA, McKeith I, Edgar C, Emre M \& Lane R 2005 Benefits of rivastigmine on attention in dementia associated with Parkinson disease. Neurology 65 1654-1656. Zhang L, Plotkin RC, Wang G, Sandel ME \& Lee S 2004 Cholinergic augmentation with donepezil enhances recovery in short-term memory and sustained attention after traumatic brain injury. Arch Phys Med Rehabil 85 1050-1055. 
Table 1. Demographic And Baseline Screening Information For All Participants.

$\begin{array}{ccc}\mathrm{AD}(\mathrm{n}=51) & \text { CONTROL }(\mathrm{n}=15) & p \\ \text { mean }(\mathrm{SD}) & \text { mean }(\mathrm{SD}) \\ \text { median (IQR) } & \text { median (IQR) }\end{array}$

\begin{tabular}{lccc}
\hline MMSE* $^{*}$ & $25(22,27)$ & $30(29,30)$ & $<0.001$ \\
AGE & $80.33(6.31)$ & $76.0(6.43)$ & 0.018 \\
GDS & $4.63(2.25)$ & $4.13(2.83)$ & 0.451 \\
YRS ED & $12(10,14)$ & $12(11,18)$ & 0.226 \\
IQ & $114.8(8.32)$ & $119.2(7.21)$ & 0.072 \\
SEX (M/F) & $25 / 26$ & $8 / 7$ & 0.769 \\
\end{tabular}

$A D=$ Alzheimer's disease group; GDS = geriatric depression scale; YRSED = years of education; IQ = premorbid IQ calculated from national adult reading test score; $M$ $=$ male, $\mathrm{F}=$ female ${ }^{*}$ data not normally distributed, therefore medians, interquartile ranges and results of Mann Whitney $U$ Tests stated; IQR = interquartile range 
Table 2. SART Results

$\operatorname{CONT}(n=15) \quad \operatorname{AD~}(n=51) \quad$ Coefficient $(\beta) \quad p$

Median (IQR) Median (IQR)

(SE)

\begin{tabular}{ccccc}
\hline Total errors & $8(3,19)$ & $29(17,45)$ & $19.75(8.71)$ & 0.027 \\
Omission & $3(0,9)$ & $19(10,33)$ & $16(6.08)$ & 0.011 \\
Commission & $4(3,6)$ & $8(3,15)$ & $1.71(2.59)$ & 0.510 \\
Correct RT & 0.474 & 0.588 & $0.109(0.062)$ & 0.083 \\
& $(0.435,0.559)$ & $(0.529,0.735)$ & & \\
Error RT & 0.467 & 0.566 & $0.073(0.055)$ & 0.188 \\
& $(0.387,0.578)$ & $(0.518,0.697)$ & & \\
\hline
\end{tabular}

$\mathrm{IQR}=$ interquartile range; $\mathrm{CONT}$ = control group; $\mathrm{AD}=$ Alzheimer's disease group; $\mathrm{SE}=$ standard error of the coefficient; $\mathrm{RT}=$ response time; Omission = omission errors; Commission $=$ commission errors; Correct $\mathrm{RT}=$ average reaction time (in $\mathrm{ms}$ ) for 4 trials preceding correct response to non-target; Error RT = average reaction time (in ms) for 4 trials preceding incorrect response to target (commission error). 
Table 3. Results of Quantile Regression Analyses of SART Errors on Cognitive task scores.

\begin{tabular}{|c|c|c|c|c|c|c|}
\hline \multirow[t]{2}{*}{$\begin{array}{l}\text { DEPENDENT } \\
\text { VARIABLE }\end{array}$} & \multicolumn{2}{|c|}{ TOTAL ERRORS } & \multicolumn{2}{|c|}{$\begin{array}{l}\text { COMMISSION } \\
\text { ERRORS }\end{array}$} & \multicolumn{2}{|c|}{$\begin{array}{l}\text { OMISSION } \\
\text { ERRORS }\end{array}$} \\
\hline & $\beta$ (SE) & $p$ & $\beta$ (SE) & $p$ & B (SE) & $p$ \\
\hline DS & $\begin{array}{l}-0.02 \\
(0.01)\end{array}$ & 0.20 & $\begin{array}{l}-0.01 \\
(0.04)\end{array}$ & 0.79 & $\begin{array}{l}-0.02 \\
(0.01)\end{array}$ & 0.17 \\
\hline SS & $\begin{array}{l}-0.02 \\
(0.01)\end{array}$ & 0.057 & $\begin{array}{l}-0.07 \\
(0.04)\end{array}$ & 0.089 & $\begin{array}{l}-0.02 \\
(0.01)\end{array}$ & 0.08 \\
\hline ADAS-COG & $\begin{array}{l}0.14 \\
(0.0)\end{array}$ & 0.004 & $\begin{array}{c}0.64 \\
(0.19)\end{array}$ & 0.003 & $\begin{array}{l}0.14( \\
0.06)\end{array}$ & 0.022 \\
\hline FLUENCY & $\begin{array}{l}-0.02 \\
(0.03)\end{array}$ & 0.49 & $\begin{array}{l}0.095 \\
(0.12)\end{array}$ & 0.44 & $\begin{array}{l}-0.03 \\
(0.04)\end{array}$ & 0.47 \\
\hline LOG MEM & $\begin{array}{l}-0.05 \\
(0.11)\end{array}$ & 0.69 & $\begin{array}{l}-0.23 \\
(0.44)\end{array}$ & 0.60 & $\begin{array}{l}-0.05 \\
(0.13)\end{array}$ & 0.69 \\
\hline PAL & $\begin{array}{c}0.00 \\
(0.01)\end{array}$ & 1.00 & $\begin{array}{c}0.00 \\
(0.02)\end{array}$ & 1.00 & $\begin{array}{c}0.00 \\
(0.01)\end{array}$ & 1.00 \\
\hline GR & $\begin{array}{c}-0.004 \\
(0.06)\end{array}$ & 0.94 & $\begin{array}{l}0.055 \\
(0.27)\end{array}$ & 0.84 & $\begin{array}{l}-0.02 \\
(0.07)\end{array}$ & 0.82 \\
\hline IADL & $\begin{array}{l}-0.01 \\
(0.02)\end{array}$ & 0.64 & $\begin{array}{l}-0.11 \\
(0.05)\end{array}$ & 0.045 & $\begin{array}{l}-0.01 \\
(0.02)\end{array}$ & 0.62 \\
\hline
\end{tabular}

$\mathrm{B}=$ regression coefficient; $\mathrm{SE}=$ standard error; $\mathrm{DS}=$ digit span; $\mathrm{SS}=$ spatial span; ADAS-Cog = Alzheimer's disease assessment scale- cognitive section; LOG MEM = logical memory 2 task. $P A L=$ Paired associates learning task; $G R=$ Grammatical Reasoning task; IADL = Instrumental Activities of Daily living task 


\section{FIGURES}

Figure 1: Boxplot Of Median Total Errors For Each Group.

Bold line = median; Box interquartile range $(I Q R)$; circles = outliers > 1.5 IQR; asterisk $=$ outliers $>3 \mathrm{IQR}$.

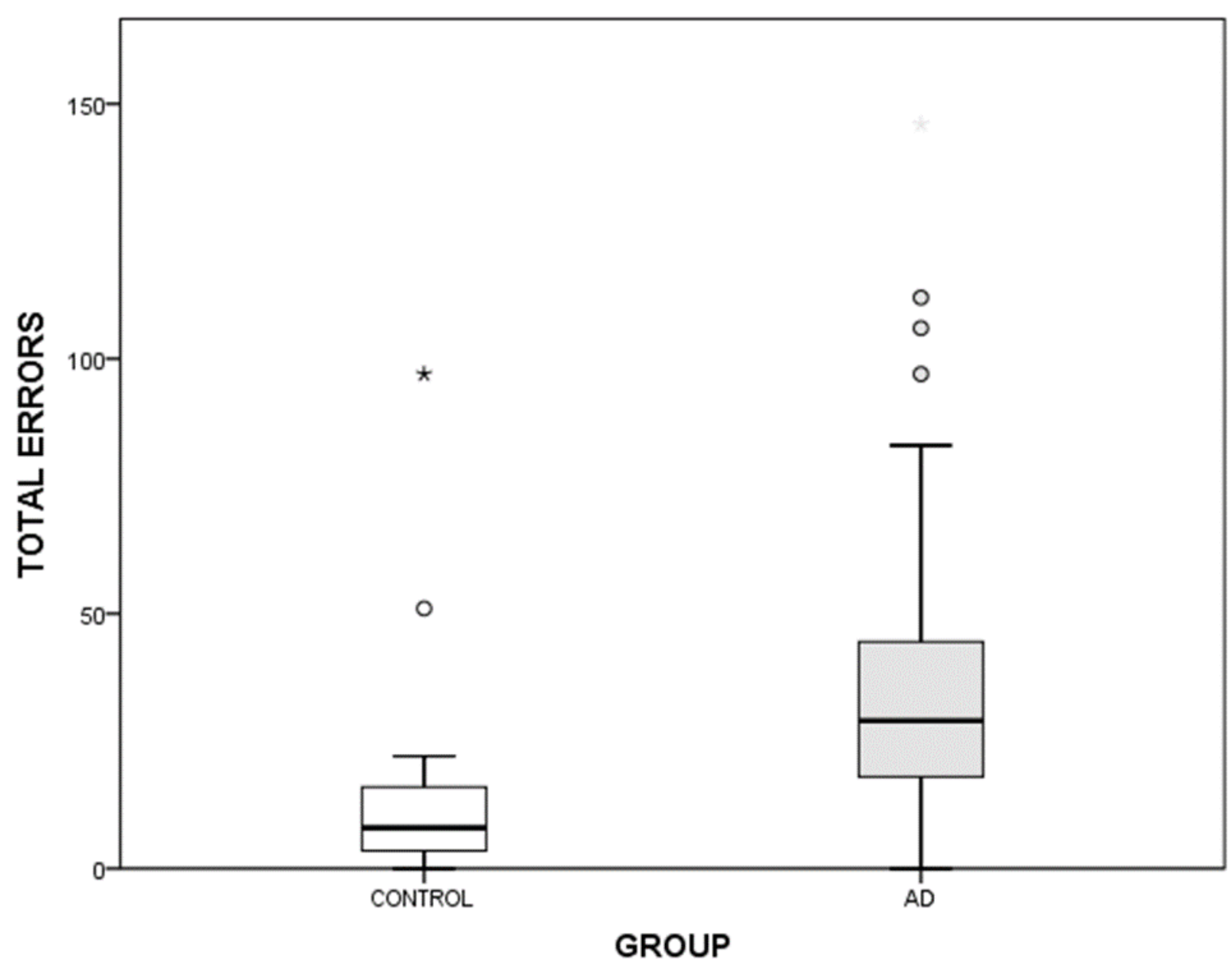


Figure 2: Correlation Between Total Errors On SART And MMSE Score.

(Spearman's rho $=-0.338, \mathrm{n}=51, p=0.015)$.

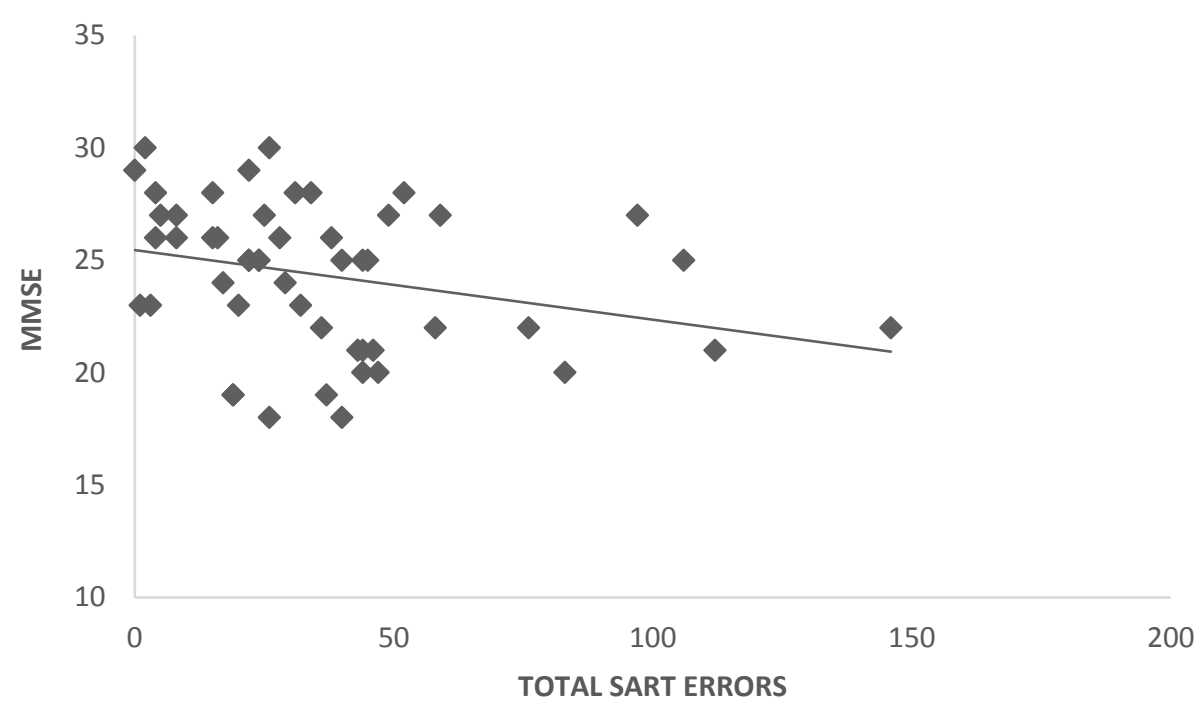

